\title{
El informe integrado: una propuesta de encaje para la información sobre capital humano
}

\author{
AINHOA SAITUA a , LOREA ANDICOECHEA ${ }^{b}$, ENEKA ALBIZUc
}

págs. 33-47

\section{HISTORIA DEL ARTÍCULO}

¿CÓMO CITAR?:

Saitua, A., Andicoechea, L. y Albizu, E. (2014). El informe integrado: una propuesta de encaje para la información sobre capital humano, Perspectiva Empresarial, 1(1). 33-47.

RECIBIDO: 11 de octubre de 2013 APROBADO: 21 de enero de 2014

CORRESPONDENCIA:

Ainhoa Saitua,

Escuela Universitaria de Relaciones Laborales (UPV/EHU) Sarriena auzoa s/n 48940, Leioa, España. la Asociación Española de Contabilidad y Administración de Empresas (AECA) sugiere que el engranaje ideal para introducir la información integrada es el Informe de gestión (AECA, 2012d) o el equivalente Management Commentary, siguiendo la terminología del International Accounting Standards Board (IASB, 2010). En este trabajo, se revisa la literatura sobre divulgación narrativa, en especial sobre recursos humanos, y una vez analizadas las recientes iniciativas normalizadoras, se elabora una propuesta acerca del posible encaje de la información sobre recursos humanos dentro del informe integrado.

PALABRAS CLAVE divulgación narrativa, información financiera, informe integrado, información no financiera, recursos humanos.

a Doctora, profesora titular de la Escuela Universitaria de Relaciones Laborales, Universidad del País Vasco, Leioa, España. Correo electrónico: ainhoa.saitua@ehu.es

b Doctora, profesora titular de la Facultad de Ciencias Económicas y Empresariales, Universidad del País Vasco, Bilbao, España. Correo electrónico: l.andicoechea@ehu.es

c Doctor, catedrático de la Escuela Universitaria de Relaciones Laborales, Universidad del País Vasco, Leioa, España. Correo electrónico: eneka.albizu@ehu.es 
¿CÓMO CITO EL ARTÍ́CULO? HOW TO CITE THIS PAPER?

\section{CHICAGO:}

Saitua, Ainhoa, Andicoechea, Lorea y Albizu, Eneka. 2014. "El informe integrado: una propuesta de encaje para la información sobre capital humano". Perspectiva Empresarial 1: 33-47.

MLA:

Saitua, Ainhoa, Andicoechea, Lorea y Albizu, Eneka. “El informe integrado: una propuesta de encaje para la información sobre capital humano". Perspectiva Empresarial 1.1 (2014): 33-47. Digital.

\section{The integrated report: A fitting proposal for information about human capital}

SUMMARY The way that companies prepare reports presents some problems (Lizcano, 2011), not only in terms of the quantity and complexity of standards, but also in terms of the quality of the corporative information that each company discloses. In short, in companies' annual reports, the obligatory information about personnel in each jurisdiction is generally limited. But in the society of current knowledge, verbal explanations about human capital acquire greater importance (Knauer, 2010; Gamerschlag and Moeller, 2011). In this context, the Integrated Reporting initiative intends to create a framework of information to satisfy the needs of a more sustainable world that includes social aspects as well as information about finance, environment and corporative governance. As principals of integrated reports it is specified that the information should be relevant, interconnected, homogenous, comparable, manageable, balanced, strategic in the short, medium and long term and verifiable (IIRC, 2012).

According to the settings posed by the International Integrated Reporting Council (IIRC), the Spanish Accounting and Business Administration Association (Asociación Española de Contabilidad y Administración de Empresas, AECA) suggests that the ideal mechanism to introduce the integrated information is in the management report (AECA, 2012) or the equivalent Management Commentary, following the terminology of the International Standards Board (IASB, 2010). In this paper the literature about narrative disclosure is reviewed, especially in relation to human resources, and once the current standard initiatives are analyzed, a proposal is elaborated about the possible exchange of human resource information within the integrated report.

KEYWORDS narrative disclosure, financial information, non-financial information, integrated report, human resources.

\section{O relatório integrado: uma proposta de encaixamento para a informação sobre capital humano}

RESUMO A maneira de informar das empresas apresenta alguns problemas (Lizcano, 2011), tanto em relação com a quantidade e complexidade de normas, quanto com a qualidade da informação corporativa que divulga cada companhia. Em concreto, nos relatórios anuais que emitem as empresas, a informação obrigatória sobre o pessoal nas diferentes jurisdições é, em geral, reduzida. Mas na sociedade do conhecimento atual, as explicações verbais sobre o capital humano adquirem maior importância (Knauer, 2010; Gamerschlag e Moeller, 2011). Em este contexto, a iniciativa Integrated Reporting pretende criar um marco de informação para satisfazer as necessidades de um mundo mais sustentável que inclua aspectos sociais junto com a informação financeira, ambiental e de governo corporativo. Como princípios dos relatórios integrados se especifica que a informação deveria ser: relevante, interligada, homogênea, comparável, manejável, equilibrada, estratégica a curto, meio e longo prazo, e verificável (IIRC, 2012).

Segundo os cenários planteados pelo International Integrated Reporting Council (IIRC), a Associação Espanhola de Contabilidade e Administração de Empresas (AECA) sugere que a engrenagem ideal para introduzir a informação integrada é o Relatório de Gestão (AECA, 2012d) ou o equivalente Management Commentary, seguindo a terminologia do International Accounting Standards Board (IASB, 2010). Em este trabalho, se revisa a literatura sobre divulgação narrativa, em especial sobre recursos humanos, e uma vez analisadas as recentes iniciativas normalizadoras, se elabora uma proposta acerca do possível encaixamento da informação sobre recursos humanos dentro do relatório integrado.

PALAVRAS CHAVE divulgação narrativa, informação financeira; informação não financeira, relatório integrado, recursos humanos. 


\section{Introducción}

En los informes anuales que emiten las empresas, la información obligatoria sobre el personal en las distintas jurisdicciones es, en general, reducida. Por una parte, en la Cuenta de pérdidas y ganancias aparecen los gastos de personal; por otra, en el Balance de situación se reflejan los créditos, deudas y provisiones relacionadas con los empleados, y en la Memoria, además de la información que explica los movimientos de los saldos de Balance y Cuenta de pérdidas y ganancias, se presenta básicamente el número y desglose de empleados. En la sociedad del conocimiento actual, el capital humano es clave para el éxito del negocio, pero la contabilidad trata a este capital humano como gasto en lugar de como activo en el balance, por lo que las explicaciones verbales resultan críticas (Knauer, 2010). Sin embargo, el área de la información narrativa sobre empleados es la que está menos desarrollada dentro de la divulgación corporativa (Mäkelä, 2010).

En los últimos años se está gestando un nuevo proyecto a nivel internacional denominado Integrated Reporting (IIRC, 2012). Se trata de un campo con mucha proyección, cuya evolución puede producir cambios, tanto en los modelos de elaboración como de revisión (auditoría financiera tradicional o aseguramiento) de la información pública (Lizcano, 2011; Moneva, 2011). Una señal de la importancia que está adquiriendo la tenemos en el Memorandum de cooperación o colaboración que firmó el IIRC con la International Federation of Accountants (IFAC) en octubre de 2012 (AIA, 2012).

Valorando positivamente el esfuerzo integrador que está realizando la iniciativa Integrated Reporting a nivel internacional, y también la Asociación Española de Contabilidad y Administración de Empresas (AECA, 2012d) con el fin de buscar el encaje de esta información dentro de las posibilidades normativas actuales, el objetivo de este trabajo consiste en actualizar la propuesta de modelo informe integrado desde una perspectiva más conceptual. Para ello, una vez que en la segunda sección se revisa la literatura académica sobre el tema y se resumen las principales corrientes normalizadoras que se han ido proponiendo con el objetivo de mejorar esta divulgación, la tercera parte se centra en las propuestas de informe integrado realizadas tanto por el IIRC como por la AECA, y es aquí donde se plantea nuestra propuesta de encaje de la información sobre recursos humanos dentro del informe integrado, finalizando con algunas reflexiones en la cuarta sección.

\section{La divulgación de información financiera y no financiera narrativa relativa a recursos humanos}

\section{Introducción}

El capital humano es clave para el éxito del negocio (Zacks, 1971; Gamerschlag y Moeller, 2011), pero la contabilidad trata a este capital como gasto en lugar de como activo en el balance. Otras formas de divulgar sobre los recursos humanos son la divulgación adicional dentro del Informe anual, en la Memoria que forma parte de las Cuentas anuales, o en el Informe de gestión que las acompaña. También puede hacerse mediante otros soportes como notas o conferencias de prensa, publicaciones voluntarias y otros canales de relaciones con inversores.

Por tanto, los esfuerzos de la empresa para lograr un personal mejor formado y más motivado pueden ser reflejados en la Memoria de las Cuentas anuales o en el Informe de gestión, en la medida que sea relevante, a partir del cambio normativo ${ }^{1}$ en transposición de directivas europeas. Pero las normas de diferentes países (Management Discussion \& Analysis-MD\&A de la Securities Exchanges Comission-SEC en Estados Unidos, etc.) han sido extremadamente prudentes, porque o bien no existe obligación de informar o, cuando es obligatorio, es el sujeto quien decide qué y cómo informar, sin que haya unas pautas adecuadas (Martínez y Burgos, 2010).

Este no es un problema específico de los recursos humanos, pues ya está siendo objeto de debate a nivel internacional en todo lo relativo a la divulgación narrativa relacionada con la información financiera (FASB, 2012; IFRS Foundation, 2013). En todo caso, en ausencia de normativa contable vigente que las obligue, las empresas suplen la falta de información sobre los recursos humanos mediante revelación voluntaria de información (García-Meca, 2008). De este modo, lo que ha cambiado en los últimos años es precisamente la oferta de información voluntaria, aumento que

1 En España, Ley 62/2003, en vigor para los ejercicios iniciados a partir del 1 de enero de 2005. 
refleja posiblemente la importancia que se le otorga al tema (Wyatt y Frick, 2010).

Los estudios sobre capital humano buscan un marco conceptual que permita formalizar mecanismos de creación de valor a partir del talento con base en la premisa de que "lo que se puede medir, se puede gestionar", pero actualmente sigue la búsqueda de un modelo general que informe sobre el capital humano (Martínez y Burgos, 2010).

El objetivo último de la contabilidad del capital humano sería asignar un valor económico al potencial humano de la empresa. Sin embargo, en el estado actual de las investigaciones se considera imposible conseguir este objetivo (pues el valor otorgado sería demasiado subjetivo), por lo que, mientras tanto, las autoras abogan por elaborar un informe de capital humano, y a partir de allí: 1) encontrar indicadores que midan aspectos clave; 2) resumir la información que ofrecen dichos indicadores, y 3) narrar los resultados de una forma coherente con la estrategia de la empresa.

Algunos resultados de las investigaciones más recientes distinguen las dos formas principales de divulgar información: la narrativa y la que se realiza por medio de indicadores. En el caso de las empresas del selectivo HDAX alemán, Knauer (2010) encuentra que el nivel de calidad de las revelaciones sobre capital humano es bajo, con aspectos considerados esenciales que ni se mencionan.

Mäkelä $(2010,2012)$ también resalta las limitaciones de las prácticas voluntarias de información social. Analizando la carta del presidente y las secciones específicas sobre temas relacionados con empleados en los informes anuales de 2008 de las 25 mayores empresas finlandesas, encuentra que la mayoría de empresas únicamente divulga una mínima cantidad de información sobre empleados, y esta información no parece seguir principios consistentes. Utilizando un enfoque interpretativo y crítico, Mäkelä analiza tanto los temas como el silencio y la retórica. Así, obtiene que la divulgación social aún refleja una imagen parcial de las personas dentro de las compañías, pues a pesar de los objetivos "emancipatorios" de la contabilidad social, los empleados se presentan de una forma estrecha y mecánica. Las empresas se reflejan como organizaciones fuertes en desarrollo continuo en la "dirección correcta", y las personas se presentan con un deseo de eficiencia para el crecimiento y el desarrollo, con un valor únicamente instrumental para la entidad.

En España, algunos estudios se enmarcaron en la línea de la Contabilidad social (Carmona y Carrasco, 1988; Pons, 1996), y otros como parte del estudio de la Responsabilidad social (Moneva y Llena, 1996; Peyró, 1997; Archel, 2003; Aranguren y Ochoa, 2008). Villacorta (2006), sobre una muestra inicial de 189 empresas de la Bolsa de Madrid en el año 2001, encontró que $14 \%$ de empresas no aportaban ninguna información voluntaria, por lo que reduce su muestra total a 163. De estas, únicamente divulgaba información voluntaria sobre empleados el 52\%. Por otra parte, la localización de la información resultaba difícil pues aparecía en diferentes lugares (en apartados como la Carta del presidente, el Informe de gestión, en información complementaria, etc.) y con diferentes denominaciones (siendo la más empleada la de "Capital humano"), razón por la que Villacorta (2006) recomienda que aparezca en apartado único, fijo y estandarizado sobre este concepto empresarial.

Por otro lado, los medios de exposición han resultado ser independientes; esto es, en ocasiones aparecen indicadores sin información literal. Con la forma literal se divulga información sobre importancia y gestión de empleados $(40 \%$ de empresas); seguridad laboral y salud (11\%) y formación (6,8\%). Así, los temas de contenido resultan limitados, por lo que propone incluir información literal sobre los esfuerzos para mantenimiento/retención, y motivación de empleados, etc. Además, se divulga con distinta profundidad, y este método literal se utiliza sobre todo para autopromocionarse, ya que incide en aspectos para mejorar su imagen. En general, se aprecia el interés de muchas empresas, en el sentido de que aparece una mayor conciencia en las grandes por ofrecer información voluntaria sobre el capital humano, pero existe una dificultad por la falta de un método establecido (Villacorta, 2006).

García-Meca y Martínez Conesa (2005) observan que cuando las empresas divulgan información sobre capital humano a los analistas financieros, lo hacen habitualmente en términos cualitativos. Los inversores pueden percibir la información cuantitativa más creíble, ya que aumenta la de verificabilidad ex post, y porque refleja más información que los inversores saben que cuesta más divulgar. Por otra parte, la información voluntaria es desigual, no homogénea (Villacorta, 2006). En todo caso, sin un marco formal para la divulgación sobre los recursos humanos, resultan problemáticas tanto su interpretabilidad como su comparabilidad (Wyatt y Frick, 2010). 
Iniciativas normalizadoras y prácticas para la mejora de la divulgación narrativa sobre recursos humanos

La literatura contable ha ido proponiendo distintas formas de incorporación de la información sobre recursos humanos desde su inclusión en los balances, hasta llegar a un sistema de indicadores en diferentes tipos de memorias narrativas. En el cuadro 1 se extractan algunas de las principales, recogidas por Sierra y Moreno (2000).

CUADRO 1. Algunas propuestas para incluir información de los RRHH en los estados financieros o en otros documentos

\begin{tabular}{|c|c|c|}
\hline ALCANCE & AUTORES & PROPUESTA \\
\hline \multirow{4}{*}{$\begin{array}{l}\text { Información } \\
\text { financiera en } \\
\text { balance }\end{array}$} & $\begin{array}{l}\text { Hermanson } \\
(1964)\end{array}$ & $\begin{array}{l}\text { Defiende la consideración de los recursos humanos de la empresa, no como } \\
\text { activos propios sino como activos operacionales, que no son propiedad de la } \\
\text { empresa pero que generan beneficios futuros, como por ejemplo la reputación } \\
\text { de la entidad, la seguridad de la demanda, la localización óptima, etc. El activo } \\
\text { recursos humanos estaría constituido por el valor de los servicios futuros que } \\
\text { se esperan obtener. Los gastos de reclutamiento constituirían un mayor valor } \\
\text { de dichos activos como gastos de adquisición, y los gastos de formalización } \\
\text { supondrían también un incremento del valor, ya que su objetivo es conseguir una } \\
\text { fuerza laboral más preparada de cara al futuro, una mejora del activo que supone } \\
\text { un mayor rendimiento. }\end{array}$ \\
\hline & $\begin{array}{l}\text { R. G. Barry } \\
\text { Corporation } \\
\text { (Zacks, 1971) }\end{array}$ & $\begin{array}{l}\text { Modelo contable implantado por esta compañía durante los ejercicios } 1969 \text { a } \\
\text { 1972, apoyado en dos nociones: la inversión en recursos humanos, que incluye los } \\
\text { gastos realizados a coste histórico con el fin de adquirir y adiestrar al personal, y la } \\
\text { amortización de la inversión en recursos humanos. }\end{array}$ \\
\hline & Marqués (1974) & $\begin{array}{l}\text { Busca una posible codificación de las variables relacionadas con el personal según } \\
\text { su consideración como recursos y como origen de fondos para la empresa. En el } \\
\text { balance, en la estructura económica aparecería la capacidad de trabajo que la } \\
\text { empresa podrá utilizar en el futuro en la generación de ingresos, y en la estructura } \\
\text { financiera, el origen de tal capacidad; es decir, la deuda con los trabajadores, } \\
\text { cuantificada como el sumatorio de todos los empleados durante su vida laboral, } \\
\text { también estimada. A diferencia del balance tradicional que busca un equilibrio } \\
\text { entre los recursos económicos y financieros derivados de transacciones pasadas } \\
\text { entre la empresa y su entorno en el momento presente, el autor defiende un } \\
\text { concepto de balance que refleje un equilibrio en el instante presente, pero en } \\
\text { términos de rendimientos esperados de sus activos y costes futuros probables de } \\
\text { sus pasivos, aunque reconoce que si bien es el mejor elaborado a efectos de toma } \\
\text { de decisiones en lo económico, no se podría utilizar para fines jurídicos y fiscales. }\end{array}$ \\
\hline & Dobija (1998) & $\begin{array}{l}\text { Propone calcular el valor del activo humano } \mathrm{V}=(\mathrm{K}+\mathrm{E})(1+\mathrm{Qt}) \text {, como el coste de } \\
\text { la vida capitalizado }(\mathrm{K}) \text {, más el coste de la educación o formación profesional } \\
\text { capitalizado }(\mathrm{E}) \text {, multiplicado por la curva de aprendizaje }(\mathrm{Qt}) \text { según los años de } \\
\text { empleo }(\mathrm{t}) \text {. En el balance quedarían los activos físicos, intangibles y financieros, } \\
\text { más los costes no expirados de reclutamiento, selección y formación de los } \\
\text { recursos humanos, financiados por el capital de los propietarios, y los activos } \\
\text { humanos, financiados con el capital de los trabajadores. }\end{array}$ \\
\hline $\begin{array}{l}\text { Otros estados } \\
\text { contables e } \\
\text { información } \\
\text { a través de } \\
\text { indicadores }\end{array}$ & $\begin{array}{l}\text { Unión Europea } \\
\text { de Expertos } \\
\text { Contables, } \\
\text { Económicos } \\
\text { y Financieros } \\
\text { (Congreso de } \\
\text { Estrasburgo, } \\
\text { 1983) }\end{array}$ & $\begin{array}{l}\text { Estructura estandarizada que incluye los siguientes puntos: } \\
\text { - Niveles de empleo } \\
\text { - Condiciones de trabajo } \\
\text { - Salud y seguridad } \\
\text { - Educación y formación } \\
\text { - Relaciones industriales } \\
\text { - Salarios y otras contraprestaciones } \\
\text { - Distribución del valor añadido }\end{array}$ \\
\hline
\end{tabular}




\begin{tabular}{|c|c|c|}
\hline ALCANCE & AUTORES & PROPUESTA \\
\hline \multirow{8}{*}{$\begin{array}{l}\text { Otros estados } \\
\text { contables e } \\
\text { información } \\
\text { a través de } \\
\text { indicadores }\end{array}$} & $\begin{array}{l}\text { El balance social } \\
\text { francés }\end{array}$ & $\begin{array}{l}\text { Desde } 1982 \text { deben emitirlo anualmente las compañías con más de } 300 \text { empleados. } \\
\text { Incluye información cuantitativa y no financiera, así como información financiera } \\
\text { sobre estos puntos (Christophe y Bebbington, 1992): } \\
\text { - Detalles de los empleados según características físicas como género y edad. } \\
\text { - Niveles de remuneración y otros gastos que sugieren la política remunerativa de la } \\
\text { empresa. } \\
\text { - Condiciones de higiene y seguridad. } \\
\text { - Otras condiciones de trabajo que influyen en las condiciones de los trabajadores, } \\
\text { como las horas de trabajo, trabajo nocturno, niveles de ruido, etcétera. } \\
\text { - Desarrollo y formación. } \\
\text { - Información sobre las relaciones entre la empresa y los empleados que indique el } \\
\text { clima social interno. } \\
\text { - Otros factores relacionados con el empleo, como las iniciativas en un estilo de } \\
\text { dirección participativo, etcétera. }\end{array}$ \\
\hline & \multirow{3}{*}{$\begin{array}{l}\text { El Informe Jenkins } \\
\text { (Aicpa, 1994) }\end{array}$} & $\begin{array}{l}\text { Se recomienda la emisión de información no financiera clasificada en ocho } \\
\text { categorías, entre las que se encuentran: }\end{array}$ \\
\hline & & $\begin{array}{l}\text { 5: Estadísticas relativas a la cantidad y calidad de los recursos clave para la empresa, } \\
\text { según el sector. Podrían ser la reserva de los recursos naturales o la edad media de } \\
\text { los empleados. }\end{array}$ \\
\hline & & $\begin{array}{l}\text { 7: Mediciones acerca de la realización y participación activa de empleados. Índices de } \\
\text { satisfacción de empleados. }\end{array}$ \\
\hline & \multirow{4}{*}{$\begin{array}{l}\text { Indicadores de } \\
\text { Sveiby (1997) }\end{array}$} & $\begin{array}{l}\text { Información que sería útil a los usuarios externos referente a la competencia, } \\
\text { estabilidad y eficiencia del personal de una empresa. }\end{array}$ \\
\hline & & $\begin{array}{l}\text { Indicadores de competencia: número de profesionales o expertos de la empresa. } \\
\text { Se refiere a las personas que diseñan, producen o presentan a sus clientes el } \\
\text { producto o el servicio requerido (no los que trabajan en funciones como contabilidad, } \\
\text { administración, etc.); número de años en la profesión; nivel de educación; formación: } \\
\text { costes de formación como porcentaje de la rotación o los días consumidos en } \\
\text { procesos de formación por profesional; medidas sobre valores y actitudes. }\end{array}$ \\
\hline & & $\begin{array}{l}\text { Indicadores de eficiencia: proporción de profesionales; beneficio por profesional; } \\
\text { valor añadido por empleado; valor añadido por profesional. }\end{array}$ \\
\hline & & Indicadores de estabilidad: edad media; antigüedad; ratio de abandono. \\
\hline
\end{tabular}

pasos de empresas españolas en medición y reporting de intangibles
Se podría optar por dos alternativas:

Uso de la memoria para informar, no solo de la medición del capital humano desde

Derksen (1996) y la perspectiva de valor, sino también comunicación de información financiera y no Johanson (1996) financiera sobre el potencial de creación de riqueza por parte de los trabajadores.

Elaboración de un estado de recursos humanos separado e independiente de las cuentas anuales.

Financiado por el Fondo Social Europeo con el objetivo de diseñar un modelo de medición y gestión del capital intelectual, cubriendo dos perspectivas, una interna (dotar a los gestores de herramientas adecuadas) y otra externa (para suministrar información a usuarios externos); fue presentado en 1997 (IUEE y KPMG Peat Marwick, 1998). Sus objetivos quedaron pendientes de cumplir por el Club Intelect, creado en 1998, y compuesto por cerca de 40 empresas (Bueno et ál., 1999).

Proyecto

Medición del $\mathrm{Cl}$

de pequeñas

y medianas

Este proyecto de la Asociación Cluster del Conocimiento únicamente pretendía crear una herramienta de medición de activos intangibles para su uso en tareas de gestión, no como emisión de información externa.

Este proyecto, iniciado en 1998 y financiado por la Unión Europea, en el que participó

España junto a Francia, Suecia, Noruega, Dinamarca y Finlandia, sí planteaba la producción de un conjunto de directrices sobre la emisión a terceros de información sobre intangibles a través de las cuentas anuales (Cañibano et ál., 1999). 
Por su parte, la revisión de Villacorta (2003) recoge modelos de gestión de los RRHH que se han aplicado en la práctica, y que se sintetizan en el cuadro 2.

CUADRO 2. Modelos de aplicación práctica de gestión del capital humano

\begin{tabular}{ll}
\hline CLASIFICACIÓN & DESCRIPCIÓN GENERAL \\
\hline & \\
& \\
& \\
& Partiendo de la experiencia \\
& del Personel Economics \\
& Institute de la Universidad de \\
Estocolmo, incluyen tendencias \\
medición por \\
competencias
\end{tabular}

\section{EJEMPLOS DE APLICACIÓN}

Bull: herramienta informática propia de $\mathrm{RH}$, dirigida a registrar la foto inicial del trabajador y sus habilidades, conocimientos y experiencias adquiridas, con el objetivo de poder situar a las personas más adecuadas en función de las líneas estratégicas de cada proyecto.

Microsoft: sistema de competencias on-line que permite: a) definir las instancias de trabajo en las que puede participar cada trabajador; b) desarrollar un ranking de empleados basado en sus competencias; c) determinar las necesidades y enlazarlas con recursos educativos orientados a fortalecer las capacidades requeridas.

Seat: plan de desarrollo de directivos cuyo principal instrumento es el Banco de Talentos, que permite disponer de información sobre los trabajadores para hacer frente al Plan de Sustituciones. Indra: Development Centre, proceso estandarizado para analizar y valorar el potencial, las experiencias y capacidades de cada trabajador, así como su posible desarrollo profesional.

Ibermática: proyecto de reorganización basado en la política de gestión de recursos humanos. La empresa identifica una serie de necesidades de formación y de desarrollo de cada empleado, y adecua el salario a su contribución a la empresa.

También BSCH, IBM, Volvo y Xerox.
Modelos de gestión por competencias
Cuanta mayor capacidad posean los empleados, mayor flexibilidad tendrá la organización en la que se integran.
Su mayor aportación es el análisis y la visualización, por medio de gráficos, del aumento de las capacidades estratégicas del personal, que se consigue cuando la empresa logra obtener de las personas el perfil pretendido, y un aumento del compromiso y de la dedicación.
Para cada uno de los aspectos del capital humano:

- vinculaciones capital humano con la estrategia

- contratación de inversiones de capital humano

- contexto para el rendimiento

- construcción y capital humano

- retención de inversores

Propone una serie de "medidas de resultados" o indicadores de la eficacia de la gestión del capital humano (por ejemplo, contribución de la contratación al fortalecimiento de capacidades) y "medidas contribuyentes" que indican el grado en que las acciones y los programas individuales respaldan las medidas de resultados (como coste de la contratación o duración del ciclo de contratación).
Proyecto T50 iniciado a finales de los años 1980 con el objetivo general de aumentar la motivación, la satisfacción de los empleados, y reducir los tiempos de producción en un $50 \%$. Su principal logro fue establecer una cuenta de pérdidas y ganancias de los recursos humanos, que se calcula en cascada por diferencia entre los ingresos totales y los diferentes costes del capital humano.
Intenta establecer otros índices para estimar el valor de los procesos (total de costos de calidad) y de los clientes (cuota de mercado), pero se centra casi exclusivamente en indicadores del capital humano por medio de los siguientes indicadores: ausencias por enfermedad, porcentaje de mujeres empleadas, tiempo de formación por empleado, gastos de formación por empleado y porcentaje de empleados con carreras universitarias. 


\begin{tabular}{|c|c|c|}
\hline CLASIFICACIÓN & DESCRIPCIÓN GENERAL & EJEMPLOS DE APLICACIÓN \\
\hline $\begin{array}{l}\text { Modelo de la } \\
\text { empresa sueca de } \\
\text { telecomunicaciones } \\
\text { Telia }\end{array}$ & $\begin{array}{l}\text { Modelo de gestión para } \\
\text { conectar los objetivos } \\
\text { estratégicos de la compañía } \\
\text { con el desarrollo de los } \\
\text { trabajadores; basa su estrategia } \\
\text { en la identificación, por parte } \\
\text { del departamento de recursos } \\
\text { humanos, de la necesidad de } \\
\text { tener empleados competentes } \\
\text { y motivados, que posean } \\
\text { además la flexibilidad necesaria } \\
\text { para desarrollar su labor en el } \\
\text { entorno futuro de constante } \\
\text { cambio. }\end{array}$ & $\begin{array}{l}\text { La gestión del capital intelectual se centra en nueve aspectos: } \\
\text { - Empleados } \\
\text { - Directivos } \\
\text { - Comunicación interna } \\
\text { - Necesidades de recursos humanos } \\
\text { - Desarrollo de competencias consideradas como factor decisivo } \\
\text { para una empresa líder } \\
\text { - Organización y métodos de trabajo } \\
\text { - Remuneración y condiciones de trabajo } \\
\text { - Ambiente de trabajo } \\
\text { Pretende elaborar un informe detallado de la composición } \\
\text { de los empleados y un documento denominado Estado de } \\
\text { los recursos humanos. En } 1995 \text { la empresa materializa una } \\
\text { contabilidad de Recursos Humanos completa (con un balance } \\
\text { de Recursos Humanos y una cuenta de pérdidas y ganancias de } \\
\text { Recursos Humanos, ampliado con anotaciones que describen los } \\
\text { cálculos realizados) paralela y complementaria a la contabilidad } \\
\text { financiera que está obligada a llevar. }\end{array}$ \\
\hline $\begin{array}{l}\text { Modelo de la } \\
\text { empresa sueca } \\
\text { SCAA (Swedich } \\
\text { Civil Aviation } \\
\text { Administration) }\end{array}$ & $\begin{array}{l}\text { Con el objetivo de ayudar a } \\
\text { la empresa a aumentar la } \\
\text { motivación y capacidad de los } \\
\text { empleados, el departamento } \\
\text { de recursos humanos intenta } \\
\text { enlazar el desarrollo de los } \\
\text { empleados con los intereses de } \\
\text { la sociedad. }\end{array}$ & $\begin{array}{l}\text { Realiza una progresiva descentralización dividiendo la empresa } \\
\text { en trece unidades para intentar conseguir los siguientes } \\
\text { objetivos: } \\
\text { - Crear una organización flexible que permita hacer frente a la } \\
\text { situación competitiva. } \\
\text { - Incrementar la competencia, productividad y eficiencia. } \\
\text { - Establecer estructuras de aprendizaje que posibiliten a cualquier } \\
\text { empleado formar parte del proceso de toma de decisiones. } \\
\text { - Crear una cultura corporativa basada en la cooperación de los } \\
\text { empleados. } \\
\text { Además elabora un balance y una cuenta de pérdidas y } \\
\text { ganancias de los recursos humanos. }\end{array}$ \\
\hline
\end{tabular}

Fuente: elaboración propia.

A partir de los años 1960 se ha tratado de medir, cuantificar y divulgar la información relativa al valor del personal como inversión, a fin de incorporarlo en los Estados financieros (CRH), y después, en conjunto con otros activos intangibles (Capital intelectual) que también ha sido objeto de normalización por AECA (2004, 2011a, 2012e). Pero vistas las dificultades de la medición y valoración, se han buscado alternativas a través de divulgación narrativa, que, además, extiende la preocupación hacia otros temas más relacionados con el comportamiento de la empresa (Contabilidad social). En la actualidad estas preocupaciones éticas/sociales y relativas al personal se engloban junto a las medioambientales (Responsabilidad social corporativa o empresarial).

De esta manera, parece que cada corriente va abarcando a la anterior, modificando ligeramente el enfoque y añadiendo nuevos temas. Sin embargo, en todas ellas aparece como problema la falta de regulación de contenidos y formatos que, a la postre, reduce la comparabilidad y, por ende, la utilidad de la información divulgada.
En todo caso, autores como Kaplan y Norton (1996) han subrayado la necesidad de interrelacionar los diferentes aspectos por considerar dentro del funcionamiento de la empresa. Por ejemplo, proponen el Cuadro de mando integral como estructura para transformar una estrategia en términos operativos. En ese sentido, la empresa debe preguntarse: ¿Cómo deberíamos aparecer ante nuestros clientes para alcanzar nuestra visión?, ¿y ante los accionistas, para tener éxito financiero?, o cómo mantener la capacidad (mediante formación y crecimiento), etc., al tiempo que se deben especificar objetivos, indicadores y acciones para cada ámbito. En un trabajo posterior, Kaplan y Norton (2004) muestran un "mapa estratégico" con las cuatro áreas: perspectiva financiera, del cliente, interna, y perspectiva de aprendizaje y conocimiento, cuyas posibles estrategias de productividad y de crecimiento tienen como finalidad última la creación de valor sostenible o a largo plazo para el accionista.

La perspectiva interna, además de gestión de operaciones, de clientes y de innovación, incluye 
los procesos reguladores y sociales, entre los que se encuentran el medio ambiente, la seguridad y salud, el empleo y la comunidad (sociedad). Por su parte, la perspectiva de aprendizaje y conocimiento incorpora tres aspectos como son: capital humano, capital de información y capital organizativo (entre los que distingue la cultura, el liderazgo, la alineación y el trabajo en equipo), todos ellos relacionados con el personal. Entendemos justificado, por tanto, que, desde este enfoque, la información sobre el personal y las capacidades del capital humano que ayudan a crear valor a la organización queden integradas con el resto de información financiera y no financiera que divulgan las empresas.

\section{Propuesta de encaje de la información sobre capital humano en el informe integrado}

En los últimos años se ha iniciado un importante debate a nivel internacional sobre el desarrollo de la denominada información corporativa integrada, lanzada por el International Integrated Reporting Committee (IIRC). Basado en el estudio La información corporativa del mañana: un sistema en riesgo, de mayo de 2011, elaborado por PwC y el Chartered Institute of Management Accountants (CIMA) junto con la Think Thank Tomorrows Company, el trabajo del IIRC elaborado con la opinión de 118 organizaciones de 22 países analiza el profundo cambio experimentado en el entorno empresarial en las últimas décadas (Lizcano, 2011).

Por un lado, los activos no financieros y los intangibles han ganado peso en el crecimiento de las compañías. Además, un gran número de compañías ha apostado por nuevas formas de creación de valor mucho más colaborativas, como la asociación con proveedores, competidores, etc. También las nuevas tecnologías han impactado entre los aspectos de la vida y, especialmente, en la capacidad de poner de manera instantánea a disposición de todo el mundo información sobre sus actividades. Finalmente, a raíz de la crisis financiera mundial, la crisis del cambio climático y la crisis de la sobreexplotación de los recursos, las empresas deben aprender a hacer más con menos.

En este contexto, los principales problemas en la manera de informar de las empresas, entonces, se pueden agrupar en cuatro grandes áreas.
Por una parte, el sistema actual de información es como un puzzle sin hacer, repleto de controles, normas y obligaciones de una complejidad tal, que ha perdido toda coherencia. Además, falta de una visión común, es decir que la calidad de la información corporativa depende en gran medida de la visión que cada compañía tiene de su utilidad: algunas lo utilizan como herramienta de marketing, otras la ven como una forma de cumplir con la legalidad, etc. Por otra parte, se observa falta de capacitación y de conocimiento. El actual nivel de complejidad que sufre el mundo de los negocios demanda nuevas capacidades para poder entenderlo, especialmente en áreas como la información no financiera, el capital intelectual o la gestión de riesgos. Por último, a la información actual se le critica que mira hacia el pasado y no hacia el futuro. Pero el informe recuerda que existe una necesidad de mirar al futuro, y de crear un sistema que nos ayude a adelantar lo que nos espera, en lugar de seguir informando del pasado (Lizcano, 2011).

El objetivo de esta iniciativa consiste en reflejar el contenido de los estados financieros, así como de la información social y medioambiental, en un único "documento" o paquete de información público, considerando los vínculos entre el desempeño financiero y de sostenibilidad, así como con el gobierno corporativo (Moneva, 2011). Para ello, el estudio de PwC señala algunos ejes sobre los que se debería construir el reporting corporativo del mañana.

Por una parte, debería centrarse en un conjunto de información que muestre la creación de valor en el largo plazo, así como los riesgos asociados. La información interna que maneja el directivo ha de estar alineada con la información que se da al exterior. Además, debería constituir un reporting con información relevante y accesible en el tiempo, que incentive a las empresas a comunicar lo que realmente es importante para su presente y para su evolución futura. Debería resultar en un sistema que ayude a la toma de decisiones de los inversores y accionistas, muestre la capacidad de las empresas de crear valor de forma sostenible, y les permita la comparación con otras compañías. Para ello, se deben incentivar el cambio y la innovación a partir de la colaboración en la confianza del sistema en todos los sectores que lo integran, y se debe lograr un mayor equilibrio de los juicios, las opiniones y el mero cumplimiento (Lizcano, 2011). 
FIGURA 1. Dimensión de complejidad de los indicadores en el modelo de Información integrada

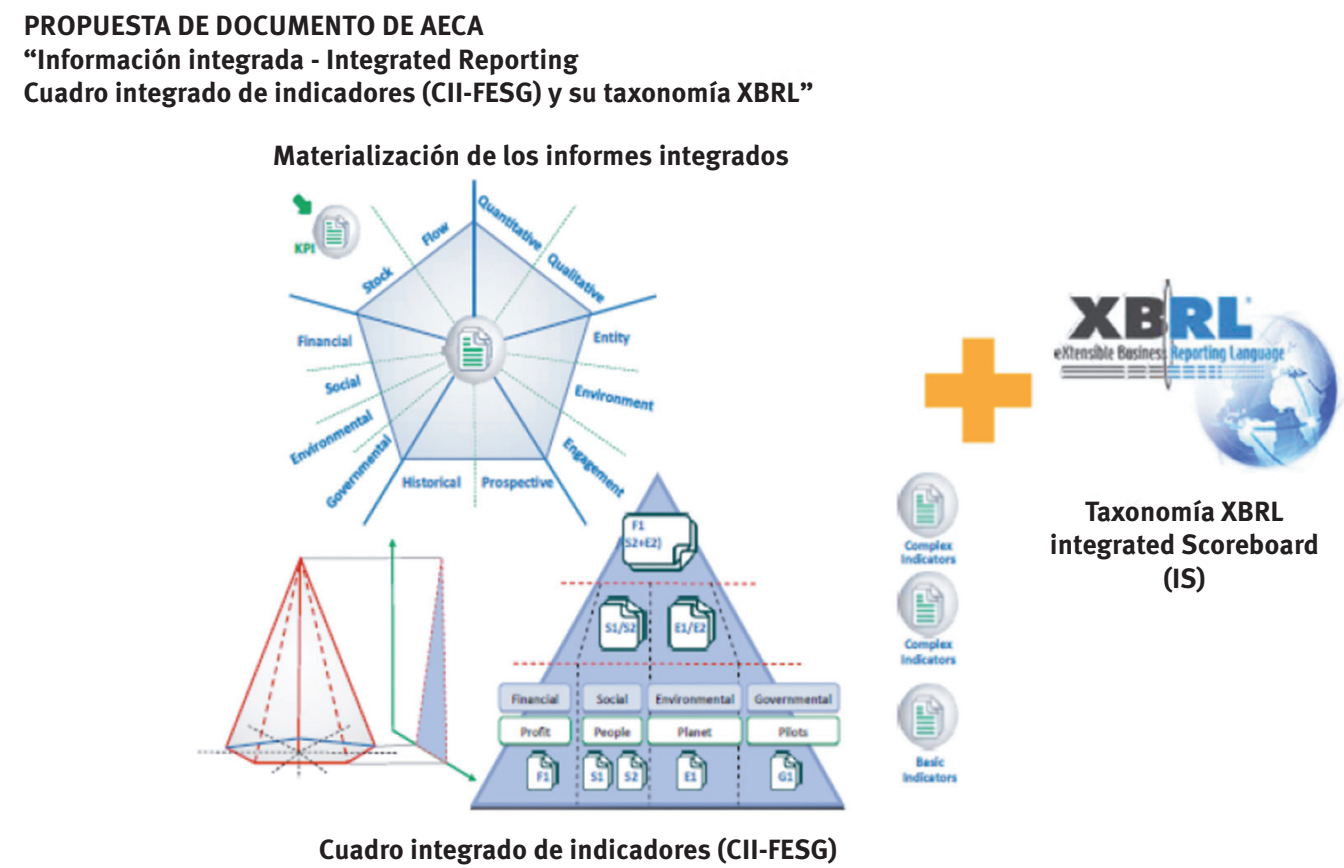

Fuente: AECA (2012d, p. 54).

En España, la Asociación Española de Contabilidad y Administración de Empresas (AECA) está desarrollando un proyecto en este sentido. En abril de 2012 se presentó la propuesta de documento AECA Información integrada-Integrated Reporting. Cuadro integrado de indicadores (CII-FESG) y su taxonomía XBRL, elaborado por tres de las diez comisiones de AECA: la de RSC, la de Nuevas tecnologías, y la de Principios y normas de contabilidad (AECA, 2012a).

Según los escenarios planteados por el IIRC $^{2}$, las propuestas para la incorporación de la Información integrada en España pasan por la introducción obligatoria del informe integrado (al estilo de Sudáfrica) o por la experimentación dentro de los requisitos legales existentes. En este contexto, la propuesta de documento de AECA sugiere que el engranaje ideal para introducir la Información integrada en el ámbito de la experimentación dentro de los requisitos legales existentes es el Informe de gestión (o equivalen-

2 En julio de 2012, el International Integrated Reporting Council (IIRC) publicó un esquema (IIRC, 2012) de lo que puede contener el marco, sobre el que esperaba emitir un borrador para someter a consulta durante 2013. te Management Commentary, siguiendo la terminología del International Accountiong Standards Board) (AECA, 2012b).

El objetivo del IIRC es crear un marco mundialmente aceptado de Información integrada que reúna información financiera, ambiental, social y de gobierno corporativo, para satisfacer las necesidades de un mundo sostenible. Y como tecnología clave del reporting integrado, el IIRC señala al XBRL, en la medida en que los enlaces de información dentro de los informes primarios facilitan el acceso a un nivel de detalle online más adecuado, allí donde sea necesario.

Como principios de los informes integrados se especifica que la información debería ser: relevante, interconectada, homogénea, comparable, manejable, equilibrada, estratégica a corto, medio y largo plazo, y verificable. Y la materialización de los informes se esquematiza visualizando la representación de la realidad empresarial mediante una pirámide pentagonal. Por una parte, los cinco lados del pentágono de la base reflejan los tipos de contenido de la información y los distintos tipos de naturaleza de esta: 1) Cuantitativa / cualitativa; 2) Entidad/Entorno / Compromiso; 3) Histórica / prospectiva; 4) Financiera /Social / Medioambiental / De gobierno; 5) Stock / Flujo. 
Tomando la pirámide de lado (en la figura 1), se tendría la vista del cuadro integrado de indicadores, CII-FESG (Financial/Environmental/Social/ Governance), que desde la base hacia arriba aumentaría su nivel de complejidad. Así, el primer "piso" estaría formado por los indicadores básicos financieros (resultado), sociales (relativo a las personas), medioambientales (referidos al planeta) y de gobierno. En el segundo piso estarían los indicadores compuestos y, en la cúspide, los complejos que relacionan distintos aspectos.

Según la propuesta de AECA (2012b), con indicadores clave de rendimiento (Key Performance Indictors - KPI) e indicadores clave de riesgos (Key Risk Indictors - KRI) se construye un cuadro integrado de indicadores formado por cinco cuadros de rendimiento, un cuadro de riesgo y 33 fórmulas, que se presentan mediante la taxonomía XBRL en un cuadro de mando integrado (Integrated Scoreboard - IS), con un doble propósito.

De una parte, la empresa que publica la información integrada, a través de la inclusión de nuevas columnas (dimensiones) o reglas de validación (fórmulas), con el fin de aumentar la complejidad de los informes por publicar, puede adaptarse al comportamiento que los inversores y administradores requieren controlar. De otra, los analistas pueden utilizar otras relaciones dimensionales o fórmulas para llevar a cabo tratamientos específicos sobre datos de la entidad, sin necesidad de incurrir en modificaciones del software. De este modo, el resultado final de la taxonomía permite a las empresas hacer un tratamiento más eficaz de la información, analizar y controlar su propio desempeño, facilitar la toma de decisiones más precisas, rápidas y de mejor calidad, y, por último, ser comparadas frente a otras, aumentando así la transparencia y la investigación en el campo de la responsabilidad social corporativa (RSC) a nivel internacional (AECA, 2012b).

En este sentido, está claro que algunas empresas pueden actuar como líderes, ofreciendo un ejemplo para otras empresas. Concretamente, las primeras entidades que publicaron su Informe integrado relativo a 2010 en España fueron la propia AECA y el banco BBVA. El grupo BBVA, que ya ha sido señalado como un referente en España a lo largo del tiempo ${ }^{3}$, es una de las 40 grandes

3 Por su balance social en los años 1980, por la información sobre capital intelectual a finales de los años 1990, por situarse en la mejor categoría de información sobre empresas de todo el mundo que fueron elegidas, resultando ser la única empresa española en el Programa Piloto del IIRC (AECA, 2011b) ${ }^{4}$. En abril de 2011 se presentó el primer informe integrado del grupo, "que supone un gran avance para insertar la responsabilidad corporativa (RC) en toda la cadena de valor de BBVA, y anticipa las tendencias más innovadoras en reporting a nivel mundial promovidas principalmente por el IIRC" (BBVA, 2011a).

A lo largo de 2010, el BBVA realizó un proceso de reflexión estratégica que contó con la participación de más de 200 directivos en siete países, con el objetivo de integrar los temas de responsabilidad corporativa (RC) en las agendas diarias de los equipos directivos. Una de las principales conclusiones fue la necesidad de impulsar las iniciativas de RC al máximo nivel, tanto local como globalmente. Por este motivo, a partir de abril de 2011 es el propio Comité de Dirección del Grupo el que ejerce la función de Comité de Responsabilidad y reputación corporativas, elevando estas cuestiones al máximo órgano ejecutivo, modelo que se replicará a nivel local en los principales países. Una de las consecuencias de esta progresiva integración fue también el nuevo modelo de reporting. De esta forma, BBVA dejó de realizar el informe de RC que venía publicando desde 2002, e integró sus indicadores sociales, medioambientales y extrafinancieros en el Informe financiero 2010, que integra plenamente la información de responsabilidad corporativa, y en cuya elaboración participaron más de mil personas. Según sus explicaciones, el diálogo permanente que se mantiene con analistas de sostenibilidad e inversores institucionales sensibles a estos temas ha sido otro factor adicional que ha motivado el dar este paso de integración del informe.

En esta línea de anticiparse a las tendencias de reporting más innovadoras a nivel mundial, además, BBVA y AECA trabajaron en el desarrollo de un estudio sobre la información integrada en el que estuvieran representadas distintas instituciones y entidades profesionales y académicas, con el fin de presentar unas conclusiones para ser trasladadas a las organizaciones públicas y privadas,

intangibles en España en 2003, según el Informe Ricardis, o por formar parte del Índice FTS4good, entre otros.

4 Posteriormente, otras empresas que participaron en el Programa Piloto del IIRC fueron Enagás, Inditex, Indra y Telefónica (AECA, 2012a). 
nacionales e internacionales. Aparte de estas empresas, las siguientes entidades españolas que elaboraron su informe integrado fueron siete: AECA (por segunda vez, y con calificación A+ de GRI), Caja Laboral, Caixabank, EDP Renovaveis, Enagas, Grupo Antena 3 e Indra (AECA, 2012c).

En el futuro, la culminación del proyecto de información integrada, en lo relativo a la obligación de presentación, entendemos que en España no tendría demasiado impacto puesto que el Informe de gestión (IG) actualmente ya acompaña a los Estados financieros. Sin embargo, sí debería tener impacto en la elección de algunos contenidos y en la forma de presentar la información en el IG, toda vez que se insiste en relacionar la explicación de los factores (incluidos los sociales) con las medidas de desempeño, así como en la medida en que se estandaricen algunos indicadores y se puedan proporcionar enlaces online.

En cambio, si bien comprendemos la propuesta de AECA (2012e) en la que se pretende que la Información integrada forme parte del Informe de gestión actual, justificado porque el contenido de este último es flexible según la regulación actual, conceptualmente creemos que debe ser al contrario. Es decir, que se debería considerar el informe integrado como un paquete completo que contenga un Informe de gestión como uno de sus elementos.

Imaginando un Balance de información no financiera que refleje la situación de la empresa, podríamos interpretar que el Capital intelectual ha querido mostrar el Activo (lo que tiene), y la corriente de Responsabilidad social se enfoca desde la obligación, el Pasivo, pero también desde la legitimidad, como un Patrimonio propio.

En todo caso, es evidente que dichos aspectos no son monetarizables (al menos, en todos sus términos) ni componen un estado de suma cero. Así, podríamos imaginar los EEFF tradicionales, complementados por otros estados no financieros (EEnoFF), pero que cumplieran con el requisito formal de presentar la información del ejercicio en curso y del precedente, a efectos comparativos. Estos EEnoFF se compondrían de los indicadores que la empresa determine relevantes a partir de un listado normalizado.

La Memoria, tanto de unos estados como de los otros, serviría a propósitos de especificación de cómo se ha elaborado la información presentada (criterios, modo de cálculo, etc.), y el Informe de gestión daría cuenta de las políticas de gestión llevadas a cabo en relación con la estrategia de la empresa, así como la evolución esperada y los planes para hacer frente a los cambios futuros.

Ante los inconvenientes de la información actual citados por Lizcano (2011), entendemos que esta propuesta ayudaría en varios sentidos. Por un lado, ante la gran dispersión de documentos y obligaciones de información actual, serviría para aglutinar toda la información relevante en un solo paquete informativo. Además, si en la actualidad la calidad de la información depende de la función que la empresa adjudique a cada tipo

FIGURA 2. Propuesta de modelo para la elaboración de un informe integrado

\begin{tabular}{|c|c|c|c|c|c|c|c|c|c|c|c|}
\hline \multirow{5}{*}{$\begin{array}{l}\text { Informe } \\
\text { Integrado }\end{array}$} & & \multicolumn{6}{|c|}{ ESTADO DE LA POSICIÓN FINANCIERA } & \multirow[b]{2}{*}{$\begin{array}{l}\text { Estado } \\
\text { de PyG }\end{array}$} & \multirow[b]{2}{*}{ ECPN } & \multirow[b]{2}{*}{ EFE } & \multirow{2}{*}{$\begin{array}{c}\text { Memoria (MEF) } \\
\text { (Notas } \\
\text { explicativas } \\
\text { de los EEFF) }\end{array}$} \\
\hline & $\begin{array}{l}\text { Estados } \\
\text { financieros }\end{array}$ & Activo & $\mathrm{X}_{1}$ & Xo & Pasivo y PN & $\mathrm{X}_{1}$ & Xo & & & & \\
\hline & \multirow[b]{2}{*}{$\begin{array}{l}\text { Estados no } \\
\text { financieros }\end{array}$} & \multicolumn{6}{|c|}{ ESTADO DE LA POSICIÓN NO MONETARIZABLE } & \multirow{2}{*}{\multicolumn{4}{|c|}{$\begin{array}{c}\text { Memoria (MENF) } \\
\text { (Notas explicativas de los EEnoFF) }\end{array}$}} \\
\hline & & $\begin{array}{l}\text { Activos intang. } \\
\text { no reconocidos } \\
\text { C Estructural } \\
\text { C Relacional } \\
\text { C Humano } \\
\text { - Diversidad } \\
\text { - Alta dirección } \\
\text { - Estabilidad } \\
\text { - Antigüedad } \\
\text { - Formación } \\
\text { - Absentismo... }\end{array}$ & $\mathrm{X}_{1}$ & Xo & $\begin{array}{l}\text { Pasivos por oblig. } \\
\text { no reconocidas } \\
\text { - Información } \\
\text { medioambiental } \\
\text { - Gobierno } \\
\text { corporativo } \\
\text { - Información } \\
\text { Social } \\
\text {... }\end{array}$ & $\mathrm{X}_{1}$ & Xo & & & & \\
\hline & \multicolumn{11}{|c|}{ Informe de gestión } \\
\hline
\end{tabular}


de documento (cumplimiento de legalidad versus función de marketing, principalmente), se promovería otorgar una misma función (comunicar el proceso de creación de valor) a toda la información divulgada, mejorando la capacitación y el conocimiento tanto de preparadores como de usuarios, por tratarse de un modelo de ámbito internacional (global), y por tanto, no local. En todo caso, la propuesta del informe integrado (IIRC, 2012) que se incorpora en nuestro modelo incluye información que mira al futuro, en respuesta a lo que viene demandando la sociedad.

\section{Conclusiones}

La reciente corriente teórica sobre divulgación de información corporativa, Integrated Reporting (informe integrado) (IIRC, 2012), pretende crear un marco mundialmente aceptado de información que incluya, entre otros, aspectos sociales, para satisfacer las necesidades de un mundo más sostenible. Se concibe como un paquete de información que incluya tanto los Estados financieros y el Informe de gestión, como el Informe de responsabilidad social corporativa.

Este artículo aporta un nuevo modelo conceptual de informe integrado en el que se busca el encaje específico para la información sobre recursos humanos. Dicho tipo de información tendría cabida tanto en la parte de Estados financieros e Informe de gestión actuales, como en la nueva de "Estados no financieros" (sobre aspectos sociales y medioambientales y de gobierno corporativo) que proponemos.

En todo caso, queda claro que en el futuro se debe mantener la atención relativa al desarrollo de las propuestas teóricas en el ámbito del Informe integrado y sus posibles incorporaciones a la regulación, y, sobre todo, relativa a las prácticas de divulgación de las empresas y la utilidad que proporcionan para los distintos usuarios, sabiendo que uno de los tipos de contenido a los que puede afectar es el relacionado con los RRHH. De modo que también se abre como posible línea de investigación futura la de conocer las opiniones relacionadas con este nuevo modelo de información por parte de los agentes involucrados en las empresas, tanto desde el punto de vista de los inversores/propietarios como principales usuarios de la información divulgada, como de los preparadores (órganos de dirección) y los empleados de las empresas.

\section{REFERENCIAS}

Association of international Accountants (AIA). (2012).

IFAC and the IIRC sign MoU on cooperation, collaboration for IR. Recuperado de http://www. aiaworldwide.com/news/779-ifac-and-the-iircsign-mou-on-cooperation-collaboration-for-irhtml American Institute of Certified Public Accountants (Aicpa). (1994). Improving Business Reporting - A Customer Focus. Meeting the Needs of Invests and Creditors. Comprehensive Report of the Special Committee on Financial Reporting.

Aranguren Gómez, N. y Ochoa Laburu, E. (2008). Divulgación de información sobre empleados y medio ambiente en España y Alemania: una nota de investigación. Revista de Contabilidad, 11(2), 123-142.

Archel Domench, P. (2003, abril-junio). La divulgación de la información social y medioambiental de la gran empresa española en el periodo 1994-1998: situación actual y perspectivas. Revista Española de Financiación y Contabilidad, XXXVIII(117), 571599.

Asociación Española de Contabilidad y Administración de Empresas (AECA). (2004, septiembre). Documento $n^{\circ} 16$ sobre Organización y sistemas: Dirección del conocimiento en las organizaciones. Madrid.

Asociación Española de Contabilidad y Administración de Empresas (AECA). (2011a, septiembre). Documento $n^{\circ} 21$ sobre Organización y sistemas: Aprendizaje organizativo. Madrid.

Asociación Española de Contabilidad y Administración de Empresas (AECA). (2011b, noviembre 3) BBVA única empresa española en el Programa Piloto del International Integrated Reporting Committee (IIRC). Lista de correo AECA 249.

Asociación Española de Contabilidad y Administración de Empresas (AECA). (2012a, abril 26). AECA presenta las primeras conclusiones de su proyecto sobre información integrada - Integrated Reporting. Nota de prensa. Madrid.

Asociación Española de Contabilidad y Administración de Empresas (AECA). (2012b, abril 26). Propuesta de documento AECA: Información integrada - Integrated Reporting. Cuadro integrado de indicadores (CII-FESG) y su taxonomía XBRL. Ponentes del documento: Francisco Flores, José Luis Lizcano, María Mora y Manuel Rejón. Presentado en Madrid.

Asociación Española de Contabilidad y Administración de Empresas (AECA). (2012c). AECA publica por segundo año consecutivo su Informe Anual Integrado y vuelve a obtener la calificación A+de GRI. Recuperado de http://www.aeca.es/temporales2012/ aeca_gri.pdf

Asociación Española de Contabilidad y Administración de Empresas (AECA). (2012d, septiembre).

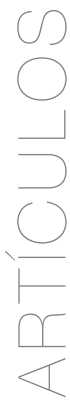


Documento $n^{\circ} 8$ RSC, $n^{\circ} 10$ Nuevas Tecnologías, $n^{\circ}$ 28 Principios y Normas de contabilidad: información integrada: el cuadro integrado de indicadores (CII-FESG) y su taxonomía XBRL.

Asociación Española de Contabilidad y Administración de Empresas (AECA). (2012e). Documento $n^{\circ} 22$ sobre Organización y sistemas: el capital intelectual de las organizaciones.

BBVA. (2011a). Presentamos nuestro primer informe integrado el 13 de abril. Recuperado de http:// bancaparatodos.com/es/presentamos-nuestro-primer-informe-integrado/

BBVA. (2011b). Presentación de nuestro informe integrado: el día después. Recuperado de http://bancaparatodos.com/videos/presentación-de-nuestro-informe-integrado-el-día-después/

Bueno, E., Rodríguez, P. \& Salmador, Y. (1999). Gestión del conocimiento y capital intelectual: análisis de experiencias. X Congreso AECA, Zaragoza.

Cañibano, L., García-Ayuso, M., Sánchez, P. \& Olea, M. (1999). Measuring intangibles to understand and improve innovation management. Preliminary results. Universidad Autónoma de Madrid.

Carmona, S. \& Carrasco, F. (1988, noviembre). Información de contenido social y estados contables. Una aproximación empírica y algunas consideraciones teóricas. Actualidad Financiera, $n^{\circ}$ 28, 2175-2192.

Christophe, B. \& Bebbington, K. J. (1992). The French Bilan Social: a pragmatic model for the development of accounting for environment? A research note. The British Accounting Review, 24(3), 281290.

Dercksen, W. (1996). Human Resource Accounting and Reporting within enterprises. White Paper, objetive 5 , Bruselas.

Dobija, M. (1998). How to Place Human Resources into The Balance Sheet? Journal of Human Resource Costing \& Accounting, 3(1), 83-92.

Financial Accounting Standards Board (FASB). (2012, julio 12). Invitation to comment. Discussion Paper. Disclosure Framework.

Gamerschlag, R. \& Moeller, K. (2011). The positive effects of human capital reporting. Corporate Reputation Review, 14(2) 145-155.

García-Meca, E. (2008, febrero). Información sobre capital humano revelada a analistas financieros. Partida Doble, 196, 76-88.

García-Meca, E. \& Martínez-Conesa, I. (2005). Assessing the quality of disclosure on intangibles in the Spanish capital market. European Business Review, 17(4), 305-313.

Hermanson, R. (1964). Accounting for human assets. Ocasional Paper $n^{\circ} 14$. East Leasing: Bureau of Business and Economic Research. Michigan State University.

IFRS Foundation (Press release, 2013, January 24). Joint effort needed to tackle disclosure problem.
Instituto Universitario Euroforum Escorial (IUEE), y KPMG Peat Marwick (1998). Proyecto Intelect. Medición del capital intelectual. IUEE: Madrid.

International Accounting Standards Board (IASB). (2010, december). Project Summary and Feedback Statement. Management Commentary. An IFRS Practice Statement.

International Integrated Reporting Council (IIRC). (2012, November 26). Integrated Reporting, Prototype Framework.

Johanson, U. (1996, December 2). Increasing the transparency of investments in intangibles. Speech given at OCDE Conference in Chateau Laurier, Otawa.

Kaplan, R. S. \& Norton, D. P. (1996). The Balance Scorecard: Translating strategy into action. Boston: Harvard Business School Press.

Kaplan, R. S. \& Norton, D. P. (2004). Mapas estratégicos. Convirtiendo los activos intangibles en resultados tangibles. Barcelona: Ediciones Gestión 2000.

Knauer, T. (2010). Relevance, quality and determinants of human capital disclosure - an empirical analysis of the German HDAX companies. Zeitschrift fur Personalforschung, 24(3), 205-230.

Lizcano, J. L. (2011, septiembre). Gobierno, responsabilidad social y transparencia: la información integrada de las empresas. Revista AECA, especial XVI Congreso, Granada.

Mäkelä, H. (2010). The narrative employee reporting in Finland. Constructing the common goal of continuous growth and development. 33 Congreso EAA, Estambul, Turquía.

Mäkelä, H. (2012). On the ideological role of employee reporting. Critical Perspectives on Accounting, 24(4-5), 360-378.

Marqués, E. (1974). La comptabilité des ressources humaines. Suresnes: Ed. Hommes et techniques.

Martínez Rey, A. \& Burgos García, M. C. (2010). La contabilidad del capital humano: lecciones aprendidas y desafíos futuros. Revista de Contabilidad y Tributación CEF, 334, 127-180.

Meritum. (1998). Measuring Intangibles to Understand and Improve Innovation Management. Proposal for a cost-shared RTD project. TSER European Programme. IV Framework Programme.

Meritum Project. (2002). Guidelines for managing and reporting on intangibles. Madrid: Fundación Arital Móvil.

Moneva, J. M. (2011, septiembre). La verificación de la información sobre responsabilidad social. Revista AECA, especial XVI Congreso, Granada.

Moneva, J. M. \& Llena, F. (1996, abril-junio). Análisis de la información sobre responsabilidad social en las empresas industriales que cotizan en bolsa. Revista Española de Financiación y Contabilidad, XXV(87), 361-401. 
Peyró Vilaplana, E. (1997). Información social y ambiental en España: un estudio empírico. IX Congreso AECA. Salamanca.

Pons, A. (1996, diciembre). La información social sobre empleados. Partida Doble, 73, 23-29.

Sierra Molina, G. J. \& Moreno Campos, I. (2000). La información contable sobre el capital humano. Partida Doble, 116, 60-71.

Sveiby, K. E. (1997). The new organizational wealth: managing and measuring knowledge-based assets. San Francisco: Berrett-Koehler Publishers.

Villacorta Hernández, M. A. (2003). Aportaciones de los modelos de gestión del capital humano a la contabilidad. Capital humano: revista para la integración y desarrollo de los recursos humanos, 16(168), 68-75.

Villacorta Hernández, M. A. (2006). Revelación de la información voluntaria sobre el capital humano en los informes anuales. Intangible Capital, 2(1), 37-71.

Wyatt, A. \& Frick, H. (2010). Accounting for investments in human capital: A review. Australian Accounting Review, 20(3), 265-273.

Zacks, G. (1971, November). People are capital investments at R. G. Barry Corporation. Management Accounting, 53(5), 53-56. 
